\title{
\begin{tabular}{l|l} 
Mibraries & DSpace@MIT
\end{tabular}
}

\author{
MIT Open Access Articles
}

\section{THE MORPHOLOGY AND DYNAMICS OF JET-DRIVEN SUPERNOVA REMNANTS: THE CASE OF W49B}

The MIT Faculty has made this article openly available. Please share how this access benefits you. Your story matters.

Citation: González-Casanova, Diego F., Fabio De Colle, Enrico Ramirez-Ruiz, and Laura A. Lopez. “THE MORPHOLOGY AND DYNAMICS OF JET-DRIVEN SUPERNOVA REMNANTS: THE CASE OF W49B." The Astrophysical Journal 781, no. 2 (January 9, 2014): L26. (C) 2014 The American Astronomical Society

As Published: http://dx.doi.org/10.1088/2041-8205/781/2/L26

Publisher: IOP Publishing

Persistent URL: http://hdl.handle.net/1721.1/93196

Version: Final published version: final published article, as it appeared in a journal, conference proceedings, or other formally published context

Terms of Use: Article is made available in accordance with the publisher's policy and may be subject to US copyright law. Please refer to the publisher's site for terms of use. 


\title{
THE MORPHOLOGY AND DYNAMICS OF JET-DRIVEN SUPERNOVA REMNANTS: THE CASE OF W49B
}

\author{
Diego F. González-Casanova ${ }^{1}$, Fabio De Colle ${ }^{1}$, Enrico Ramirez-Ruiz ${ }^{2}$, and Laura A. Lopez ${ }^{3,4,5}$ \\ ${ }^{1}$ Instituto de Ciencias Nucleares, Universidad Nacional Autónoma de México, A. P. 70-543, 04510 D. F., Mexico \\ ${ }^{2}$ Department of Astronomy and Astrophysics, University of California, Santa Cruz, CA 95064, USA \\ ${ }^{3}$ MIT-Kavli Institute for Astrophysics and Space Research, 77 Massachusetts Avenue, 37-664H, Cambridge, MA 02139, USA \\ Received 2013 September 24; accepted 2013 December 11; published 2014 January 9
}

\begin{abstract}
The circumstellar medium (CSM) of a massive star is modified by its winds before a supernova (SN) explosion occurs, and thus the evolution of the resulting supernova remnant (SNR) is influenced by both the geometry of the explosion as well as the complex structure of the CSM. Motivated by recent work suggesting the SNR W49B was a jet-driven SN expanding in a complex CSM, we explore how the dynamics and the metal distributions in a jet-driven explosion are modified by the interaction with the surrounding environment. In particular, we perform hydrodynamical calculations to study the dynamics and explosive nucleosynthesis of a jet-driven SN triggered by the collapse of a $25 M_{\odot}$ Wolf-Rayet star and its subsequent interaction with the CSM up to several hundred years following the explosion. We find that although the CSM has small-scale effects on the structure of the SNR, the overall morphology and abundance patterns are reflective of the initial asymmetry of the SN explosion. Thus, we predict that jet-driven SNRs, such as W49B, should be identifiable based on morphology and abundance patterns at ages up to several hundred years, even if they expand into a complex CSM environment.
\end{abstract}

Key words: hydrodynamics - ISM: individual objects (W49B, G43.3-0.2) - ISM: supernova remnants - methods: numerical - nuclear reactions, nucleosynthesis, abundances

Online-only material: color figures

\section{INTRODUCTION}

The morphology and dynamics of young supernova remnants (SNRs) depend on the geometry of the explosions as well as the distribution of the circumstellar medium (CSM; see review by Vink 2012). SNRs from massive progenitors are luminous from the interaction of the ejecta with nearby gas that was expelled by the progenitor system (Weaver et al. 1977), probably during a red supergiant (RSG) stage. Beyond the relic red giant wind material, the CSM is likely to have been highly disturbed by previous evolutionary stages (Garcia-Segura et al. 1996; Ramirez-Ruiz et al. 2005) as well as by density discontinuities or gradients in the interstellar medium (ISM; Dohm-Palmer \& Jones 1996), such as molecular cloud edges. Consequently, the relative role of the explosion and the environment in shaping SNRs remains an outstanding question (Canizares 2004).

The difficult task of unraveling the origin of asymmetries in SNRs is best illustrated by W49B (G43.3-0.2), the most luminous SNR in our Galaxy in X-rays $\left(L_{\mathrm{X}} \sim 10^{38} \mathrm{erg} \mathrm{s}^{-1}\right.$; Immler \& Kuntz 2005) and in $\gamma$-rays (Abdo et al. 2010). X-ray imaging of W49B has shown an unusual morphology, with a highly elliptical shape comprised of an iron-rich bar with two plumes at its edges (Hwang et al. 2000; Miceli et al. 2006; Lopez et al. 2009). The integrated X-ray spectrum exhibits strong emission lines from several metals (e.g., $\mathrm{Si}, \mathrm{S}, \mathrm{Ar}, \mathrm{Ca}$, and Fe: Hwang et al. 2000; Keohane et al. 2007) with supersolar abundances, indicating an ejecta origin. These metals appear segregated, with the $\mathrm{Fe}$ absent in the east, while the silicon and other intermediate-mass elements are distributed more homogeneously (Lopez et al. 2009, 2013b). Two scenarios have been proposed to explain the anomalous morphology of W49B: a bipolar/jet-driven core-collapse supernova (CC-SN; Keohane

\footnotetext{
4 NASA Einstein Fellow.

5 MIT Pappalardo Fellow in Physics.
}

et al. 2007; Lopez et al. 2013b) or a spherically symmetric SN that expanded into an inhomogeneous ISM (Zhou et al. 2011).

Observational evidence shows that W49B has a complex environment: near-infrared imaging revealed bright [Fe II] rings thought to be wind material from a massive star progenitor (Keohane et al. 2007). Additionally, ${ }^{13} \mathrm{CO}$ and warm, shocked $\mathrm{H}_{2}$ gas have been detected surrounding the SNR (Simon et al. 2001; Keohane et al. 2007). However, the abundance ratios of the ejecta from across W49B are similar to the predictions for a jet-driven explosion of a $25 M_{\odot}$ Wolf-Rayet (WR) progenitor (Maeda \& Nomoto 2003) and are inconsistent with values expected in symmetric CC-SNe (Lopez et al. 2013b). Moreover, the distinct morphology of Fe is likely due to anisotropic ejection of heavy metals, since Fe Xxv line emission is missing from the eastern part of the SNR, even though the temperatures there are sufficient to produce that line emission.

These observations show that the X-ray line emission morphology of young SNRs like W49B can be altered not only by the structure of the ejecta but also by the spatial distribution of the elements synthesized within the SN explosion. Numerical models of jet-driven SN explosions (e.g., Khokhlov et al. 1999) show that the greater kinetic energy at the poles of the exploding star can lead to efficient synthesis of nickel there, while lower$Z$ elements are ejected more isotropically (e.g., Mazzali et al. 2005). The issue of how this bipolar morphology is modified by the expansion into the external medium (Lopez et al. 2009) and its implications for W49B remains an unresolved problem.

In this Letter, we aim to determine the degree to which the dynamics and abundance structures of a jet-driven SNR are modified by the interaction with the external medium. As the CSM of a massive star is modified by their winds, we consider shocked wind bubbles for the surrounding media. Given that a complete simulation of a jet-driven SNR's evolution is computationally expensive, we perform two separate hydrodynamical calculations, each equipped to describe the behavior of the 
bipolar outflow at two different epochs. In Section 2, we present a brief description of the numerical methods. In Section 3, we use a simulation with a general equation of state (EOS) and a reaction network to determine the nucleosynthesis that accompanies a jet propagating through the massive star. In Section 4, we employ the resulting models (i.e., the density, velocity, and compositional structure of the ejecta) as the initial conditions in the calculation of the subsequent hydrodynamical expansion of the SNR and the associated thermal X-ray emission produced by the hot shocked gas. Finally, in Section 5 we summarize our results and present our conclusions.

\section{NUMERICAL METHODS}

In order to study the dynamics and explosive nucleosynthesis of a jet-driven $\mathrm{SN}$, we have performed a set of two-dimensional cylindrically symmetric simulations. We use the adaptive mesh refinement code Mezcal (De Colle \& Raga 2006; De Colle et al. 2008, 2012a, 2012b), which incorporates a nuclear network module and different EOSs. The Helmholtz EOS (Timmes $\&$ Swesty 2000), which includes radiation, ion pressure, and electron-positron degeneracy pressures, is used in Section 3 when calculating the propagation of the jet through the star. The nucleosynthesis is calculated by coupling the hydrodynamics equations to an inexpensive, seven element $\alpha$-chain nuclear network (Timmes et al. 2000), comprising the evolution of the ${ }^{4} \mathrm{He},{ }^{12} \mathrm{C},{ }^{16} \mathrm{O},{ }^{20} \mathrm{Ne},{ }^{24} \mathrm{Mg},{ }^{28} \mathrm{Si},{ }^{56} \mathrm{Ni}$ mass densities and energy losses due to neutrino cooling. In the nuclear network, the ${ }^{28} \mathrm{Si}$ and ${ }^{56} \mathrm{Ni}$ mass fractions are representative of the silicon and iron mass fraction groups, respectively. A detailed description of the implementation of the nuclear burning module in the Mezcal code is presented in Holcomb et al. (2013).

When computing the long-term evolution of the SNR, we perform two simulations. First, we assume that the energy losses through X-ray line emission are small so that the dynamics of the flow are not affected by gas cooling and use an adiabatic index $\gamma=5 / 3$. Second, our code was extended with a subroutine to calculate the ionization age of ejecta elements assuming collisional equilibrium, where the cooling function for gas of temperature $T$ with metallicity $Z$ is taken from Gnat \& Sternberg (2007) for $T>10^{4} \mathrm{~K}$.

\section{A JET-DRIVEN SUPERNOVA MODEL FOR W49B}

Based on the abundances derived from the X-ray spectra of W49B, Lopez et al. (2013b) showed the nucleosynthesis was consistent with a bipolar explosion of a $25 M_{\odot}$ WR star. Thus, for our initial conditions, we adopt the $E 25$ pre-SN stellar model of Heger et al. (2000). The pre-SN star, initially a $25 M_{\odot}$ WR star, has a mass of $5.45 M_{\odot}$, a radius of $R_{*}=0.43 R_{\odot}=$ $3 \times 10^{10} \mathrm{~cm}$, and an iron core mass of $1.45 M_{\odot}$ extending out to a radius $R_{\mathrm{Fe}}=2.2 \times 10^{8} \mathrm{~cm}$. A uniform jet with sharp edges and opening angle of $\theta_{\mathrm{j}}=30^{\circ}$ is injected from a boundary located at $R_{\mathrm{Fe}}$ with a velocity $v_{\mathrm{j}}=0.3 \mathrm{c}$. The jet injection time is $\Delta t=6 \mathrm{~s}$, which results in a total energy of $6 \times 10^{51} \mathrm{erg}$.

The results of the simulation of the jet-driven $\mathrm{SN}$ explosion are presented in Figure 1, where the mass density $\rho$, pressure $P$, silicon $X(\mathrm{Si})$ and nickel $X(\mathrm{Ni})$ mass fractions of the expanding collimated ejecta are plotted $10^{2} \mathrm{~s}$ after the jet has broken free from the star. In the direction of the propagation of the jet, the resulting post-shock temperatures are high (with $T \gtrsim 5 \times 10^{9} \mathrm{~K}$ ), leading to complete oxygen and silicon burning there (Maeda \& Nomoto 2003; Maeda \& Tominaga 2009).

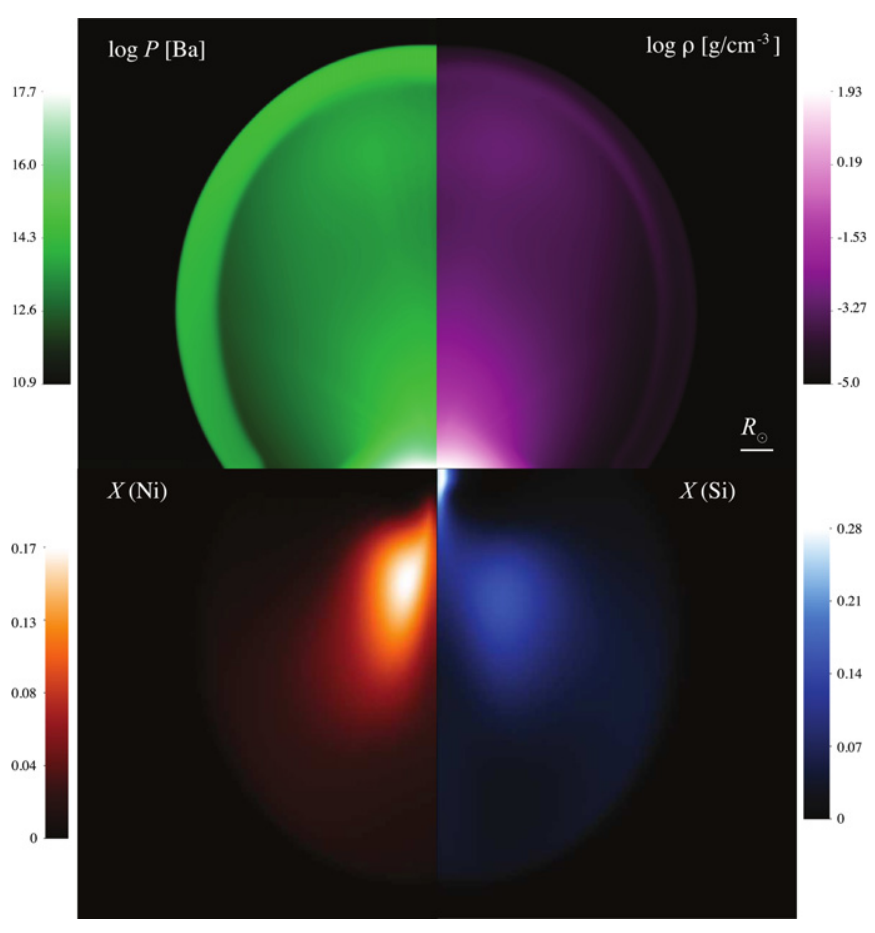

Figure 1. Jet-driven SN explosion at the onset of the homologous phase, which is achieved $100 \mathrm{~s}$ after the bipolar ejecta has broken free from the $R_{*}=0.43 R_{\odot}$ stellar progenitor. The pressure $P$, mass density $\rho$, and the silicon $X(\mathrm{Si})$ and nickel $X(\mathrm{Ni})$ mass fractions are indicated in each frame with corresponding size scales in units of $R_{\odot}$. The jet propagation inside the WR progenitor is calculated using an adaptive cylindrical grid of physical size $l_{\mathrm{r}}=l_{\mathrm{z}}=4.5 \times 10^{10} \mathrm{~cm}$, with $10^{2} \times 10^{2}$ cells on the coarsest grid and seven levels of refinement, which corresponds to a maximum resolution of $7 \times 10^{6} \mathrm{~cm}$.

(A color version of this figure is available in the online journal.)

Consequently, the expansion velocity of the newly synthesized ${ }^{56} \mathrm{Ni}$ is closely aligned with the jet axis.

As it expands inside the progenitor star, the jet is unable to move the stellar material at a velocity comparable to its own and hence is abruptly slowed down. A large fraction of the energy output during this phase is accumulated into a cocoon surrounding the jet (e.g., Ramirez-Ruiz et al. 2002). Thus, the lateral production of ${ }^{56} \mathrm{Ni}$ is confined to the deepest stellar layers, and elements ejected in this direction by the cocoon's expansion are primarily the products of hydrostatic nuclear burning with some explosive oxygen-burning products (e.g., Si, S). As a result, the distribution of nickel is much more elongated than that of silicon (Maeda \& Nomoto 2003; Maeda \& Tominaga 2009), as can be clearly seen in the abundance distributions shown in Figure 1. The jetted outflow, responsible for synthesizing ${ }^{56} \mathrm{Ni}$, is observed to carry more energy and inertia than the broader (incomplete silicon and oxygen burning) component, so that the latter never overtakes it. Therefore, the distribution asymmetry in the abundance is expected to be preserved as the $\mathrm{SN}$ material sweeps up the external medium.

\section{THE EVOLUTION OF A YOUNG JET-DRIVEN SNR}

Next, we consider the interaction of the jet-driven SNR with the CSM. Since the radiative cooling time for the optically thin gas at an age $\leqslant 1000 \mathrm{yr}$ is greater than the age of the remnant, we see no appreciable change between the adiabatic and the radiative cooling simulation runs. Support abounds in the literature for the notion that efficient cosmic ray acceleration can impact the evolution a of a SNR (Caprioli \& Spitkovsky 


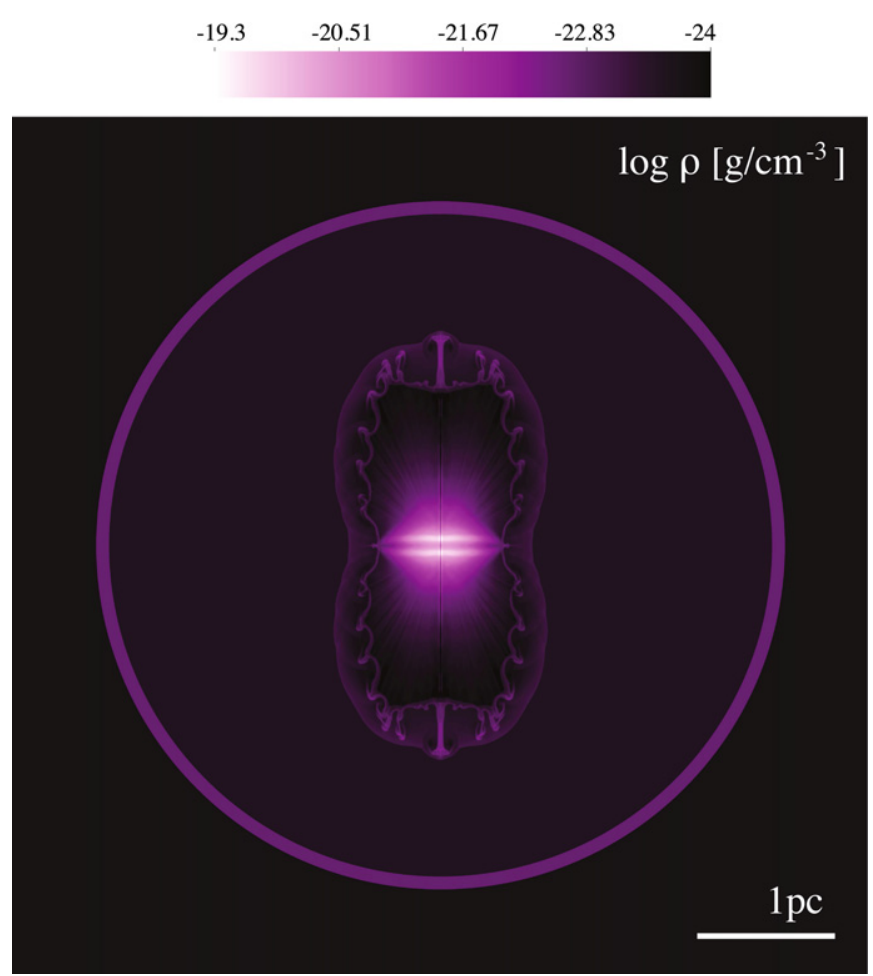

Figure 2. Evolution of a jet-driven SN remnant inside the wind bubble structure expected around a $25 M_{\odot}$ massive star for the case of an ISM pressure typical of the high-density molecular region surrounding W49B. The dense RSG shell, with a mass of about $6.5 M_{\odot}$, is located at $2.5 \mathrm{pc}$. The region outside the red supergiant shell is the remains of the bubble from the main-sequence phase while the density in the shocked region is approximately constant. The output of the hydrodynamic simulations at the time of homology (Figure 1) was mapped onto the grid at small spatial scales. Shown is the logarithm of the mass density at an evolutionary time of $20 \mathrm{yr}$ after the supernova explosion. The calculations are performed on a grid with size $l_{\mathrm{r}}=l_{\mathrm{z}}=5 \times 10^{19} \mathrm{~cm}$, with $10^{2} \times 10^{2}$ cells on the coarsest grid and seven levels of refinement.

(A color version of this figure is available in the online journal.)

2013, and references therein). However, there is not a simple prescription that could be used to simulate the energy losses by cosmic rays at shock fronts. Recent attempts to model efficient particle acceleration by globally adjusting the adiabatic index of the fluid (e.g., Warren \& Blondin 2013) have shown that although the stability of the contact discontinuity depends on the compressibility of the fluid, the overall dynamics are not appreciably affected by cosmic ray energy losses.

The pre-SN stellar wind depends on the evolutionary stages prior to and during the WR phase. A standard evolutionary track at solar metallicity (e.g., Garcia-Segura et al. 1996) is for an O star to evolve through a RSG phase or luminous blue variable phase with considerable mass loss (e.g., Ramirez-Ruiz et al. 2001), and then to become a WR star. At low metallicity and for some binary stars, the RSG phase may be absent (Izzard et al. 2004). When the fast WR wind starts blowing, it sweeps up the RSG wind material into a dense, cold shell (Chevalier et al. 2004; Georgy et al. 2013). The radius of the shocked region depends on the duration of the WR phase $t_{\mathrm{wr}}$, the ratio of the mass-loss rates $\dot{M}_{\mathrm{wr}} / \dot{M}_{\mathrm{rsg}}$, and wind velocities $v_{\mathrm{wr}} / v_{\mathrm{rsg}}$. Here we assume $t_{\mathrm{wr}}=3 \times 10^{4} \mathrm{yr}, \dot{M}_{\mathrm{wr}} / \dot{M}_{\mathrm{rsg}}=10^{-2}$ and $v_{\mathrm{wr}} / v_{\mathrm{rsg}}=10^{2}$ (Maeder \& Meynet 1994).

With these reference values, the radius of the wind termination shock is $R_{\mathrm{t}}=0.3 \mathrm{pc}$ and the pressure (in units of $\mathrm{Ba} / k$, where $k$ is the Boltzmann constant) in the shocked region is $P_{\mathrm{s}} / k=2.5 \times 10^{4} \mathrm{~K} \mathrm{~cm}^{-3}$ (Chevalier \& Imamura 1983). If the external pressure $P_{0}>P_{\mathrm{s}}$ (as expected in molecular clouds where $P_{0} / k=10^{5} \mathrm{~K} \mathrm{~cm}^{-3}$ : Blitz 1993), the expansion of the RSG will be stalled. The density in the shocked region is approximately constant, and beyond the contact discontinuity (at $R_{\mathrm{c}} \approx 3 R_{\mathrm{t}}$ : Chevalier \& Imamura 1983), a region of shocked (dense) cold RSG material is expected.

The CSM around a $25 M_{\odot}$ massive stellar progenitor appears as in Figure 2. At solar metallicity, the star has a long-lived RSG phase that dramatically affects the surrounding medium. In particular, a dense RSG shell occurs at roughly $2.5 \mathrm{pc}$, approximated here for the case of a high ISM pressure typical of molecular clouds. We note that in reality, the RSG shell is subject to hydrodynamical instabilities (Garcia-Segura et al. 1996), and its radius depends sensitively on several parameters (Chevalier $\&$ Imamura 1983; Georgy et al. 2013), including $t_{\mathrm{wr}}$ (which varies with mass and metallicity), $v_{\mathrm{wr}} / v_{\mathrm{rsg}}, \dot{M}_{\mathrm{wr}} / \dot{M}_{\mathrm{rsg}}$, and $P_{0}$. In addition, many of these parameters can be significantly altered by the rotation of the progenitor star (e.g., Ekström et al. 2012).

Before the SNR size becomes comparable to the scale length of the RSG shell, the morphology of the remnant will reflect the geometry of the initial explosion (Ramirez-Ruiz \& MacFadyen 2010). This can be seen by comparing the density contours in Figures 1 and 2. The expansion of the bipolar remnant will be stalled when it reaches the sharp density discontinuity at $\sim 2.5$ pc. The interaction of the ejecta with the RSG shell results in the disruption of the dense thin layer before the jet undergoes substantial lateral expansion. Consequently, the initial asymmetry of the explosion is preserved (Figure 3). The RSG shell, clearly apparent in Figure 2, will be pushed outward at high velocities as a result of the interaction. Figure 3 also shows the development of fingers extending from the deformed shell and produced by the Rayleigh-Taylor instability. Despite the large density contrast, the pressure in the remnant remains fairly uniform. Both the deformed thin layer and the protruding fingers expand at velocities $\approx 500 \mathrm{~km} \mathrm{~s}^{-1}$. These large velocities are produced as a result of successive shocks ramming into the deformed thin layer. The strands of material formed as the thin dense layer is disrupted could be responsible for the prominent X-ray line emission filaments observed in W49B (Keohane et al. 2007; Lopez et al. 2013b).

The accompanying evolution of the metal distribution is shown in Figure 4. A broadening of the iron-group filaments is clearly seen after the interaction with the RSG shell, which is accompanied by the development of small-scale density fluctuations. After $t=260 \mathrm{yr}$ and radii of $r \approx 3 \mathrm{pc}$, the irongroup $\left(X_{\mathrm{Ni}}\right)$ and silicon $\left(X_{\mathrm{Si}}\right)$ structures have become highly irregular, with the iron-group elements being significantly more extended along the jet axis. The remnant also shows a complex velocity structure with fast-moving iron filaments expanding along the explosion center, which are less pronounced in the initially more centrally concentrated silicon-rich material. Thus, although the structure of the CSM plays a prominent role, our results demonstrate that the measurable properties of young SNRs depend sensitively on the initial distribution of the metalrich ejecta.

\section{DISCUSSION}

The results presented here illustrate how the dynamics of young, jet-driven SNRs, are influenced by the interaction of the ejecta with the CSM. By comparing our hydrodynamical simulations to the observational properties of $\mathrm{W} 49 \mathrm{~B}$, we can gain a deeper understanding on the nature of bipolar SNR. A few observables are relatively easy to determine from 


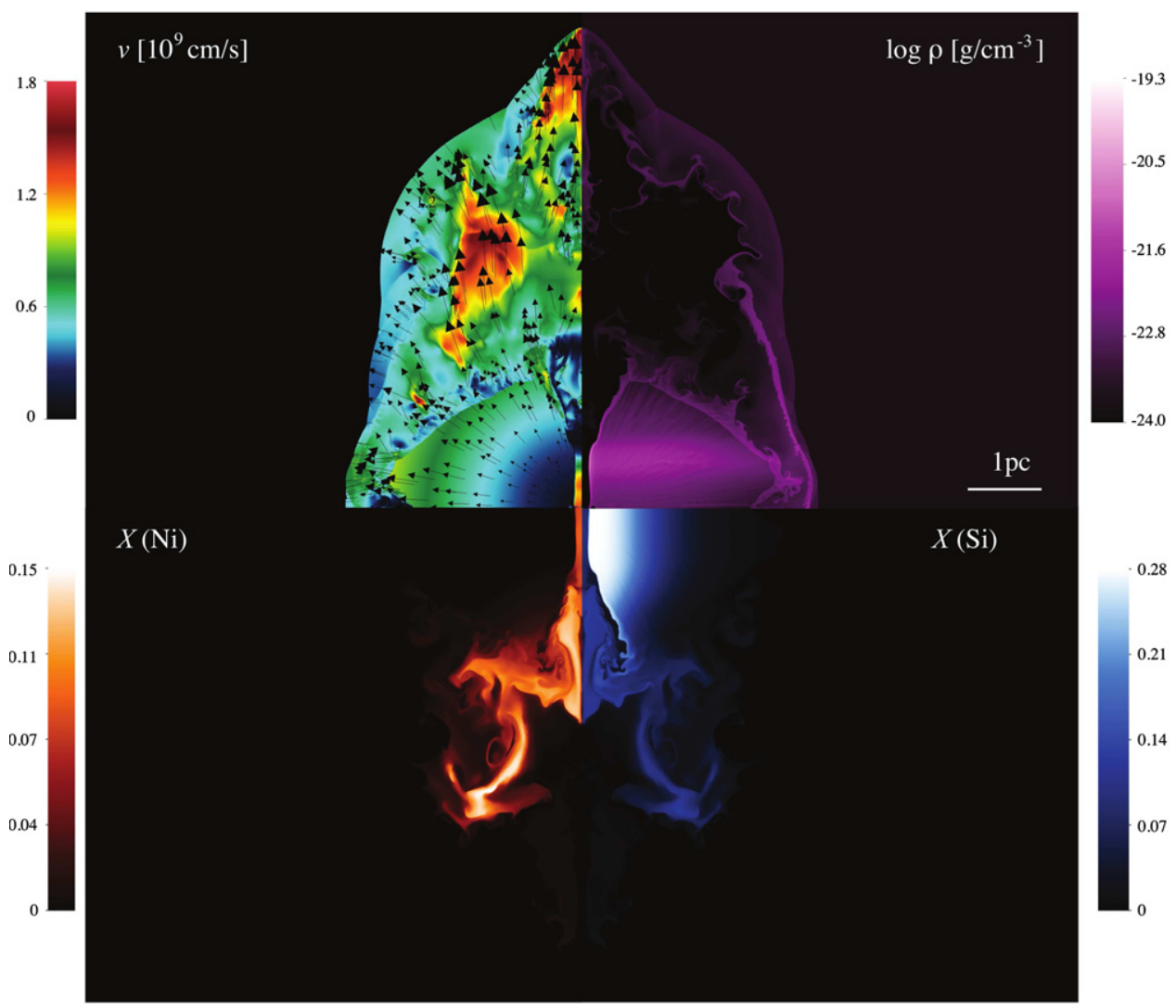

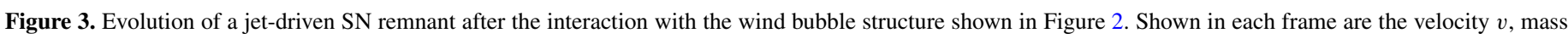

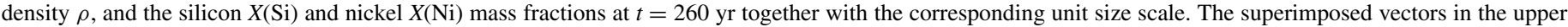
left frame show the velocity field of the resulting SN remnant.

(A color version of this figure is available in the online journal.)

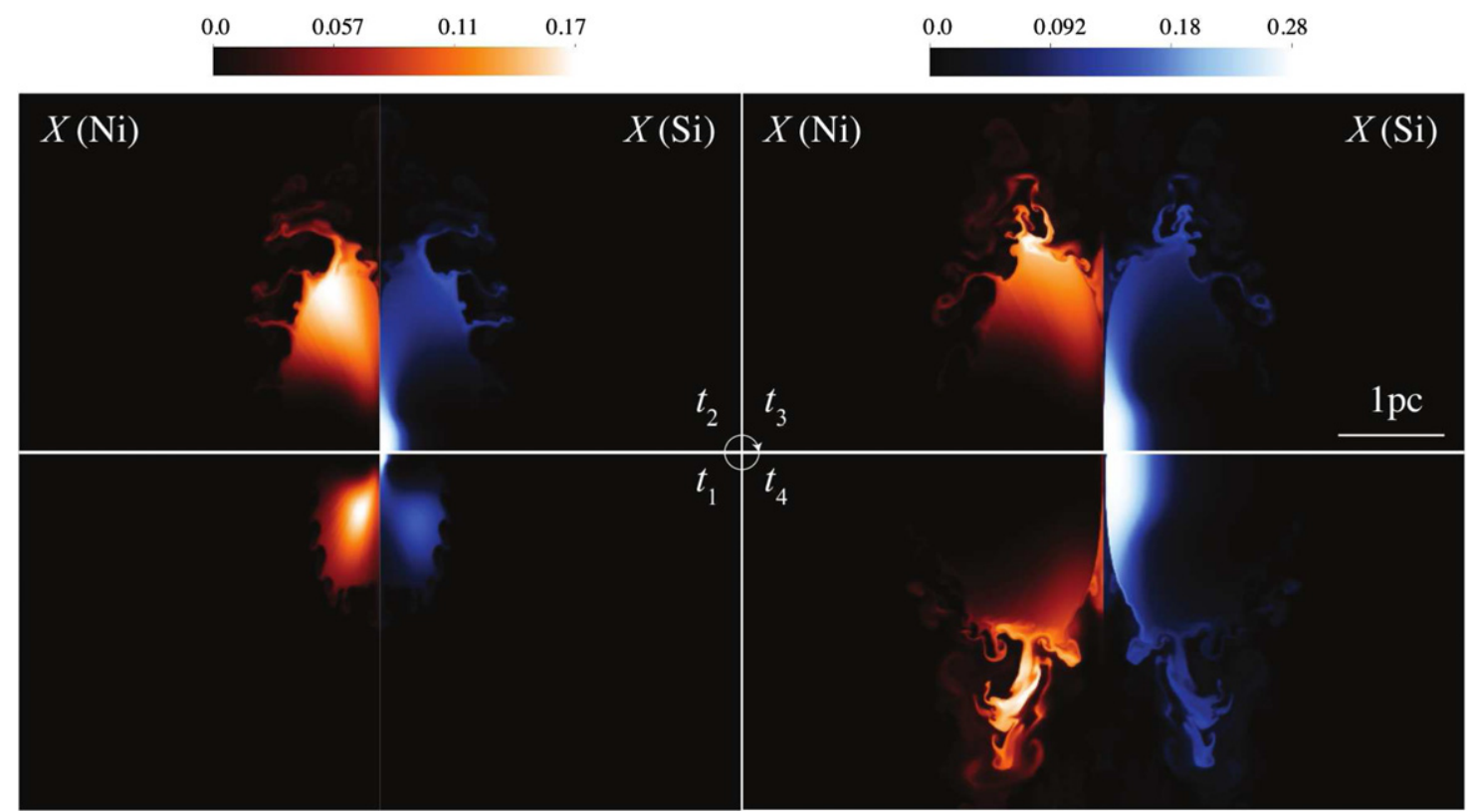

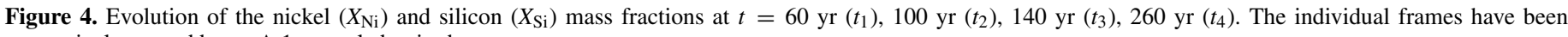
successively rotated by $\pi$. A 1 pc scale bar is shown.

(A color version of this figure is available in the online journal.)

one-dimensional hydrodynamical models, e.g., expansion rates and thickness of flow structures. However, other comparisons can be more challenging. For example, to predict the distribution of the observed thermal X-ray emission, multidimensional simulations are necessary to follow the detailed distribution and composition of ejecta, their clumping, and the inhomogeneous CSM (e.g., Badenes et al. 2003).

To date, simulations to reproduce the thermal X-ray morphology of W49B have not been done due to an absence of a commonly accepted dynamical model. The observed anisotropic 


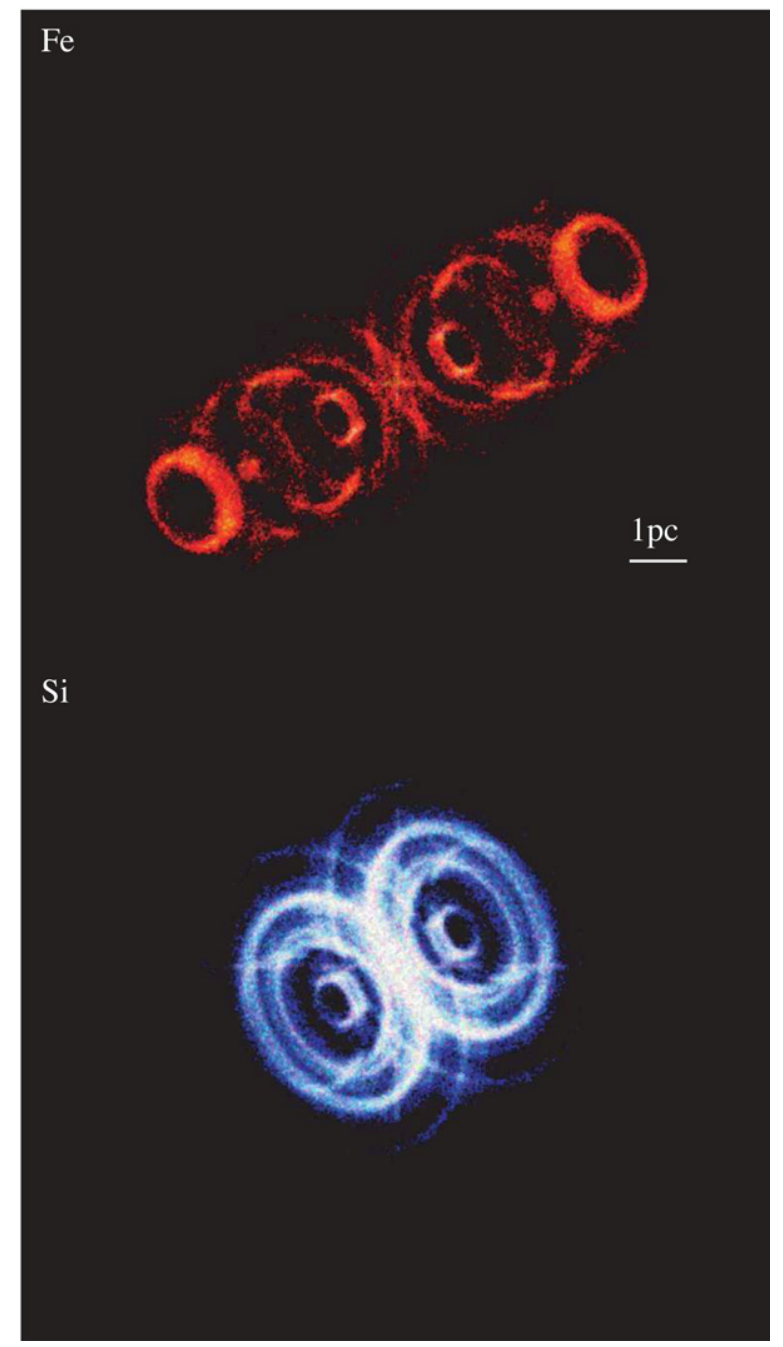

Figure 5. Fe XXV and Si XIV X-ray emission maps. The maps are obtained integrating the respective emission coefficients along the line of sight assuming collisional ionization equilibrium (Lopez et al. 2009). The $200 \times 200$ pixel images are computed at an evolutionary age of $700 \mathrm{yr}$, which is comparable to the estimated dynamical age of W49B (Pye et al. 1984). To match the characteristics of the $\mathrm{W} 49 \mathrm{~B}$, the results of the simulation have been rotated $45^{\circ}$ with respect to the plane of the sky (in the direction of the observer), and $120^{\circ}$ with respect to the $z$-axis (the initial direction of the jet), before the emission being integrating along the line of sight. The size of the line emitting region is comparable to the observed size of W49B, which at a distance of $8 \pm 2 \mathrm{kpc}$ is $\approx 4^{\prime}$.

(A color version of this figure is available in the online journal.)

distribution of strong X-ray line features from $\mathrm{Si}$ and $\mathrm{Fe}$ in W49B strongly suggests that heavy-element-enriched material was produced asymmetrically deep within the exploded star (Lopez et al. 2013b). The calculations presented here support this idea. Figure 5 shows that even in the presence of a uniform external medium, the thermal X-ray morphology of a jet-driven SNR is dominated by a bright iron jet, rimmed by a plateau of explosive oxygen-burning products, such as silicon and sulfur. The bright X-ray luminosity of W49B may be characteristic of the interaction with the CSM produced by extensive mass loss prior to the explosion (Figure 2). This CSM has a mass comparable to that of the SN material and is not expected to spread out beyond a few parsecs in length because of the large surrounding pressure. Only after several centuries, the SN ejecta will break free from this relatively dense CSM (Figure 4).

But W49B is also interacting with a molecular cloud in the east (Simon et al. 2001; Keohane et al. 2007). Detailed studies of the X-ray surface brightness along its circumference presented by Lopez et al. (2013a), revealed the stalling of the expansion in the eastern region as the ejecta collides with molecular material. This interaction is likely responsible for halting the expansion of the jetted ejecta, creating the observed thermal X-ray wing feature from the lateral expansion of shocked material (Lopez et al. 2013a, 2013b). As relativistic ions are accelerated through this dense molecular material by highly supersonic shocks, the resultant pion decay is likely to be responsible for producing the observed $\mathrm{GeV} \gamma$-rays (Abdo et al. 2010). In the future, three-dimensional simulations incorporating an anisotropic external medium will be necessary to test our two-dimensional results and to thoroughly investigate the effects of the molecular cloud interaction on the resulting thermal X-ray structures.

Based on the absence of an X-ray luminous compact object, Lopez et al. (2013b) predicted a black hole was formed during the explosion of W49B. If it originated from a jet-driven CC-SN, it could have been one with a sub-relativistic collimated outflow, as opposed to the rare, high Lorentz factor jets in gamma-ray bursts (Gehrels et al. 2009). For example, it could be the common jet-driven SN discussed by Khokhlov et al. (1999), in which case the core collapse continues to form a black hole instead of halting upon producing a neutron star.

Even for the simplest CSM distribution, we have shown that the structure resulting from the expansion of a jet-driven remnant like W49B is considerably different from that of a more standard CC-SN explosion. In particular, the morphology at early stages (after several hundred years) still reflects the asymmetry of the explosion, even in a complex, dense CSM (Figure 1). In principle then, one could identify jet-driven SNRs based on the abundance distribution and morphology, although the CSM influences their overall shape somewhat, especially at late times.

We thank D. Castro, G. Garcia-Segura, A. MacFadyen, R. Margutti, S. Pearson, A. Soderberg, and the referee for insightful discussions. We acknowledge support from the David and Lucile Packard Foundation, NSF grant AST-0847563, and the DGAPAPAPIIT-UNAM grant IA101413-2. Support for L.A.L. was provided by NASA through the Einstein Fellowship Program and the MIT Pappalardo Fellowship in Physics.

\section{REFERENCES}

Abdo, A. A., Ackermann, M., Ajello, M., et al. 2010, ApJ, 722, 1303 Badenes, C., Bravo, E., Borkowski, K. J., \& Domínguez, I. 2003, ApJ, 593, 358 Blitz, L. 1993, in Protostars and Planets III, ed. E. H. Levy \& J. I. Lunine (Tucson, AZ: Univ. Arizona Press), 125

Canizares, C. R. 2004, in Recent X-ray Observations of Supernova Remnants, ed. A. C. Fabian, K. A. Pounds, \& R. D. Blandford (Cambridge: Cambridge Univ. Press), 107

Caprioli, D., \& Spitkovsky, A. 2013, ApJ, 765, L20

Chevalier, R. A., \& Imamura, J. N. 1983, ApJ, 270, 554

Chevalier, R. A., Li, Z.-Y., \& Fransson, C. 2004, ApJ, 606, 369

De Colle, F., Granot, J., López-Cámara, D., \& Ramirez-Ruiz, E. 2012a, ApJ, 746,122

De Colle, F., \& Raga, A. C. 2006, A\&A, 449, 1061

De Colle, F., Raga, A. C., \& Esquivel, A. 2008, ApJ, 689, 302

De Colle, F., Ramirez-Ruiz, E., Granot, J., \& Lopez-Camara, D. 2012b, ApJ, 751,57

Dohm-Palmer, R. C., \& Jones, T. W. 1996, ApJ, 471, 279

Ekström, S., Georgy, C., Eggenberger, P., et al. 2012, A\&A, 537, A146

Garcia-Segura, G., Langer, N., \& Mac Low, M.-M. 1996, A\&A, 316, 133

Gehrels, N., Ramirez-Ruiz, E., \& Fox, D. B. 2009, ARA\&A, 47, 567

Georgy, C., Walder, R., Folini, D., et al. 2013, A\&A, 559, A69

Gnat, O., \& Sternberg, A. 2007, ApJS, 168, 213

Heger, A., Langer, N., \& Woosley, S. E. 2000, ApJ, 528, 368

Holcomb, C., Guillochon, J., De Colle, F., \& Ramirez-Ruiz, E. 2013, ApJ, 771,14 
Hwang, U., Petre, R., \& Hughes, J. P. 2000, ApJ, 532, 970

Immler, S., \& Kuntz, K. D. 2005, ApJ, 632, L99

Izzard, R. G., Ramirez-Ruiz, E., \& Tout, C. A. 2004, MNRAS, 348, 1215

Keohane, J. W., Reach, W. T., Rho, J., \& Jarrett, T. H. 2007, ApJ, 654, 938

Khokhlov, A. M., Höflich, P. A., Oran, E. S., et al. 1999, ApJ, 524, L107

Lopez, L. A., Pearson, S., Ramirez-Ruiz, E., et al. 2013a, ApJ, 777, 145

Lopez, L. A., Ramirez-Ruiz, E., Castro, D., \& Pearson, S. 2013b, ApJ, 764, 50

Lopez, L. A., Ramirez-Ruiz, E., Pooley, D. A., \& Jeltema, T. E. 2009, ApJ, 691,875

Maeda, K., \& Nomoto, K. 2003, ApJ, 598, 1163

Maeda, K., \& Tominaga, N. 2009, MNRAS, 394, 1317

Maeder, A., \& Meynet, G. 1994, A\&A, 287, 803

Mazzali, P. A., Kawabata, K. S., Maeda, K., et al. 2005, Sci, 308, 1284

Miceli, M., Decourchelle, A., Ballet, J., et al. 2006, A\&A, 453, 567

Pye, J. P., Becker, R. H., Seward, F. D., \& Thomas, N. 1984, MNRAS, 207, 649
Ramirez-Ruiz, E., Celotti, A., \& Rees, M. J. 2002, MNRAS, 337, 1349

Ramirez-Ruiz, E., Dray, L. M., Madau, P., \& Tout, C. A. 2001, MNRAS, 327,829

Ramirez-Ruiz, E., García-Segura, G., Salmonson, J. D., \& Pérez-Rendón, B. 2005, ApJ, 631, 435

Ramirez-Ruiz, E., \& MacFadyen, A. I. 2010, ApJ, 716, 1028

Simon, R., Jackson, J. M., Clemens, D. P., Bania, T. M., \& Heyer, M. H. 2001, ApJ, 551, 747

Timmes, F. X., Hoffman, R. D., \& Woosley, S. E. 2000, ApJS, 129, 377

Timmes, F. X., \& Swesty, F. D. 2000, ApJS, 126, 501

Vink, J. 2012, A\&ARv, 20, 49

Warren, D. C., \& Blondin, J. M. 2013, MNRAS, 429, 3099

Weaver, R., McCray, R., Castor, J., Shapiro, P., \& Moore, R. 1977, ApJ, 218,377

Zhou, X., Miceli, M., Bocchino, F., Orlando, S., \& Chen, Y. 2011, MNRAS, 415,244 